\title{
Trial Sets Parameter
}

National Cancer Institute

\section{Source}

National Cancer Institute. Trial Sets Parameter. NCI Thesaurus. Code C117463.

A character or string that represents the long name of the trial set parameter. 